\title{
Planet dispersal in binary systems during transient multiple star phases
}

\author{
F. Marzari ${ }^{1}$ and M. Barbieri ${ }^{2}$ \\ 1 Dipartimento di Fisica, University of Padova, Via Marzolo 8, 35131 Padova, Italy \\ e-mail: marzari@pd.infn.it \\ 2 LAM, Traverse du Siphon, BP 8, Les Trois Luc, 13376 Marseille Cedex 12, France \\ e-mail: mauro.barbieri@oamp.fr
}

Received 14 May 2007 / Accepted 31 May 2007

\begin{abstract}
Aims. If a significant fraction of binary star systems spent some time as inclined triple systems, either during their formation process or as the outcome of several close dynamical encounters in a crowded stellar environemnt, then the number of planets in binaries would be significantly lower than around single stars. The stellar chaotic phase preceding the instability of the triple system and the wide oscillations in eccentricity and inclination of the companion star due to the high mutual inclination between the companion and the singleton would quickly eject planets orbiting the binary in S-type orbits.

Methods. We perform numerical simulations of the dynamical evolution of hierarchical triple star systems with planets hosted around the primary star of the inner binary. Different values of mutual inclination, binary separation and singleton initial semimajor axis are explored in a statistical way.

Results. We find that a significant mutual inclination $i_{\mathrm{m}}$ between the singleton and the binary is a key factor for instability of the planetary system. When $i_{\mathrm{m}}$ is larger than $\sim 40^{\circ}$ the fraction of planets in the binary surviving the chaotic phase of the triple declines dramatically. The combination of eccentricity and inclination oscillations of the binary companion induced by the secular perturbations of the singleton and the sequence of close encounters preceding the ejection of one star fully destabilize a planetary system extending beyond $1 \mathrm{AU}$ from the star. For $i_{\mathrm{m}}$ around $90^{\circ}$ the percentage of surviving planets is lower than $20 \%$ for all binaries with a semimajor axis smaller than $200 \mathrm{AU}$.

Conclusions. The frequency of planets in binaries with low separation may be strongly reduced by the residence of the pair in the past in a temporary inclined hierarchical triple.
\end{abstract}

Key words. methods: $N$-body simulations - stars: planetary systems

\section{Introduction}

Over 65 percent of the main sequence stars in the solar neighborhood are members of binary or multiple star systems (Duquennoy \& Mayor 1991). As a consequence, answering the question of whether planets can form and persist near one of the stars in a binary has far-reaching implications for the overall frequency of planetary systems. Studies on the long term stability of planets in binaries have shown that a planet cannot be located too far away from the host star or its orbit will be destabilized by the gravitational perturbations of the companion star. Holman \& Wiegert (1999) found that the stable/unstable boundary depends on the mass ratio and eccentricity of the binary, but for a wide range of parameters stable orbits may extend well beyond one tenth of the binary semimajor axis. However, in terms of the probability of finding a planet in binary systems, the dynamical stability analysis is not exaustive since it does not take into account the profund influence that stellar dynamic interactions may have had on the early evolution of a planetary system in a binary.

It has been suggested that most binaries originate from the decay of multiple systems (Reipurth 2000; Larson 1972, 1995, 2001; Kroupa 1995). The most common configuration among multiple systems is the hierarchical triple, where a singleton orbits around the baricenter of a binary system. A hierarchical triple can become unstable after some time, depending on its initial orbital parameters, leading to the disintegration of the triple (Eggleton \& Kiseleva 1995; Kiseleva et al. 1996). This disintegration occurs via a phase of chaotic evolution whose outcome is the ejection of one of the three stars (typically the least massive body) on an unbound trajectory. The other two stars, members of the original binary, are left in a more tightly bound binary. In a previous paper (Marzari \& Barbieri 2007, herein after MB1) we showed that the orbital changes of the binary and the strong gravitational perturbations during the chaotic phase prior to the singleton ejection can influence the final configuration of a planetary system hosted by the primary star of the pair. However, in the context of near-coplanarity between the binary and the singleton, planets can survive the triple decay in most cases and adapt to the new orbital parameters of the binary. The major effect would be a significant change in the orbital configuration of the system after the triple instability with respect to the original configuration, as an outcome of the planet formation process.

In this paper we consider the dynamic effects of the decay of inclined hierarchical triples on planetary systems. In particular, we will focus on planet survival during the unstable triple configuration. At present, determinations of the mutual inclinations of the two orbits in hierarchical triple stellar systems are available only for a very limited number of cases and are often ambiguous. Fekel (1981) determined that at least $1 / 3$ of a sample of 20 triple star systems have an inclination exceeding 
$15^{\circ}$ and are not coplanar. Sterzik \& Tokovinin (2002) analysed a different set of 22 visual triples finding an average mutual inclination of $79^{\circ}$. However, in both the studies the mutual inclination was derived from incomplete observational data. To compute unambiguous mutual inclinations for triples, both radial velocities and visual orbits are required for the inner and outer system. So far, only six nearby systems have been observed with both methods and have direct and precise measured orbits (Muterspaugh et al. 2006). The values of mutual inclination for these systems range from $24^{\circ}$ to $132^{\circ}$ but the sample is too small to give hints on the real distribution of inclinations among triples. The mutual inclination of triples may either be primordial and related to the formation process of the triple by fragmentation of a molecular cloud or it may form at later times because of dynamical interactions, like encounters, between single stars and binaries in a dense cluster-like environment. In the latter case the inclination is due to the encounter geometry between the binary and the single star and should be randomly distributed. Any deviation from randomly oriented orbits may be an important indication of the relative importance of the two formation mechanisms. Assuming that planets can form in the binary before the bound hierarchical triple becomes unstable, the dynamical interactions between the stars during the chaotic phase can strongly affect the stability of the planetary system.

We can envision two different scenarios for planet formation and subsequent destabilization within an inclined hierarchical triple:

- A primordial binary star system forms in a star cluster and planets accumulate from a circumstellar disk around the main star either by core-accretion (Pollack et al. 1996) or by disk instability (Boss 1997). The existence of a few gas giant planets in binary systems with separation of a few tens of $\mathrm{AU}$ ( $\gamma$ Cephei and GL 86) suggests that the perturbations of the companion star are not strong enough to prevent the formation of planets in binaries (Thébault et al. 2006; Boss 2006). Successively, a temporary hierarchical triple builds up because of dynamical interactions between the primordial binary, with planets, and a passing by singleton or binary (Ford et al. 2000). In a dense stellar environment with a large abundance of binaries this is a frequent event (McMillan et al. 1991). In the presence of a significant inclination between the singleton and the binary orbit, the planetary system in the binary is strongly destabilized during the transitional triple state by both the secular perturbations of the singleton and the frequent stellar encounters during the chaotic phase preceding the break up of the triple into a binary and singleton. The destruction of the triple may occur either because it is unstable and after a short timescale the singleton escapes or because of an encounter with other objects, single stars or binaries. After the ejection of the singleton in a hyperbolic orbit, the primordial binary has different orbital parameters but it is also depleted, in most cases, of its original planetary system.

- A primordial inclined hierarchical triple forms by fragmentation of a single, rotating, dense molecular cloud (Boss 1988). Planets can grow on S-type orbits around the primary star of the binary, possibly by disk instability (Boss 1997). Selfgravitating density clumps can contract into planets in only a few hundred years. It is unrealistic to expect that planets can form by core-accretion in this scenario because of the strong secular perturbations that the singleton would apply on a long timescale on the secondary star and then, indirectly, to a putative planetesimal disk around the binary (assuming that planetesimals could form in such a highly perturbed circumstellar disk). Even in this case, the combined destabilizing effects of secular perturbations and stellar encounters in the chaotic phase preceding the ejection of the singleton in a hyperbolic orbit destabilize the planetary system around the primary.

In this paper we numerically model the orbital evolution of planets in S-type orbits in a binary, member of an inclined unstable hierarchical triple. We consider different mutual inclinations between the binary and the singleton, while in MB1 we simulated only the planar case. We find that, contrary to the low inclined cases, only in a limited number of cases do planetary systems extending beyond $1 \mathrm{AU}$ survive after the chaotic phase of stellar encounters when the mutual inclination between singleton and companion is larger than $\sim 40^{\circ}$. There are two mechanisms that, acting in synergy, destabilize planets around the primary star.

- A large initial mutual inclination between the outer stars excites consistent eccentricity-inclination oscillations of the binary companion with periods of the order of some thousand years and more. These oscillations, well described by a quadrupole-level secular theory (Mazeh \& Shahan 1979; Ford et al. 2000), strongly affect the orbits of the planets around the primary, forcing most of them to leave the system on hyperbolic orbits. In the phase of high eccentricity, the companion moves closer to the planetary system, reducing the region of stability (Holman \& Wiegert 1999) and perturbing the planetary orbits. After a few Kozai-cycles a large fraction of the planetary system is destroyed. For retrograde orbits the dynamics is more complex but the evolution is still characterized by wide oscillations of both eccentricity and inclination. This kind of Kozai mechanism in stellar triples is different to that described by Malmberg et al. (2007) where the secular interactions involved only the companion star of an isolated binary and the planets.

- The second mechanism giving the "coup de grace" to the planetary system is the sequence of close encounters between the singleton and the companion star of the binary occurring during the chaotic phase preceding the disgregation of the triple. Close encounters between stars on mutually inclined orbits are much more effective in destabilizing the planets than in the low inclination case.

Both the eccentricity oscillations of the secondary star and the close encounters conspire against the survival of a planetary system around the main star of the binary. If indeed binary stars are born as triple or higher multiplicity stellar systems or they are temporarily involved in unstable triples, their planetary systems would be fully destabilized in most of the inclined cases. The fraction of planets in binaries observed at present would than be lower than that around single stars. The number of binaries depleted of planets would depend on the distribution of mutual inclination in the primordial triples. If coplanarity dominates, as might be the case if the majority of temporary triples originated directly from the fragmentation of an interstellar cloud (Boss 1988), then the binaries stripped of their planets would be a minority. On the other hand, if most of the triples formed by gravitational interactions in a dense stellar environment, the large mutual inclinations would lead to a strong planet depletion among the surviving binaries. In this scenario, it is an important observational challenge to increase the statistics of known triple systems with unambigous determination of the mutual inclination. At present only about $15 \%$ of planets have been found in 
multiple stellar systems. This is probably an observational selection effect in favor of single target stars but it might also be a first indication that binary systems are depleted of planets by the past violent dynamical evolution of the stars.

We will not explore in this paper the full complexity of the hierarchical triple dynamics as performed in Ford et al. (2000). We are interested on the consequences of the large variations of the star orbital elements on the planets and we perform statistical numerical simulations giving the fraction of planets surviving the chaotic phase of unstable triples. We also do not investigate the planetary formation process in detail, but we assume that planets can form by either of the two mechanisms, core-accretion or gravitational instability.

In Sect. 2. we describe the numerical model adopted for the numerical integration of the trajectories of the stars and planets. Section 3 is devoted to the statistical analysis of the survival of planets in S-type orbits around the primary star. In Sect. 4 we present our conclusions.

\section{The model}

Our numerical model consists of 3 stars, two locked in a binary system and the third orbiting the barycenter of the pair. A set of 10 massless bodies started on circular orbits around the primary star simulate a planetary system that formed in the early phases of evolution of the binary. The semimajor axes of the test bodies are regularly spaced from 1 to $10 \mathrm{AU}$ and the initial inclinations are all set to $0^{\circ}$ with respect to the binary orbital plane. The trajectories of the stars and of the "planets" are computed with the numerical integrator RADAU (Everhart 1985). It handles in a very precise manner close encounters between massive bodies and it does not require a fixed hierarchical structure such as HJS (Beust 2003) or SYMBA5 (Duncan et al. 1998).

To model the outcome of the triple instability in all possible configurations is a difficult task since the parameter space to explore is wide. For this reason we select a limited number of parameters to be sampled while the others are left unchanged. To better compare our results with those presented in MB1, we adopt the same masses for the stars i.e. 1 and 0.4 solar masses for the binary, 0.4 solar mass for the singleton. An eccentricity of 0.2 is adopted for both the binary and the singleton, taking into account that the orbit of the singleton is defined with respect to the barycenter of the binary. The mutual inclination is sampled between $0^{\circ}$ and $180^{\circ}$ including in this way retrograde orbits of the singleton. For any value of the semimajor axis of the binary $a_{\mathrm{b}}$, we sample different values of the semimajor axis of the singleton $a_{\mathrm{s}}$ and of the orbital angles other than those giving the mutual inclination. For any set of $\left(a_{\mathrm{b}}, a_{\mathrm{s}}, i_{\mathrm{m}}\right)$ we perform 20 simulations with random initial orbital angles to increase the statistics on the star and planet dynamical behaviour.

\section{The dynamical sources of instability}

In this section we discuss in detail the two mechanisms leading to destabilization of a putative planetary system extending beyond $1 \mathrm{AU}$ around the primary star of a binary in an inclined temporary triple. In Fig. 1 we show the evolution of a model with $a_{\mathrm{b}}=70 \mathrm{AU}, a_{\mathrm{s}}=212 \mathrm{AU}$ and initial mutual inclination $i_{\mathrm{m}}=90^{\circ}$. In this configuration, the critical semimajor axis for long-term stability of planetary orbits around the primary is, according to Holman \& Wiegert (1999), around 21 AU. Our initial planetary system, extending out to $10 \mathrm{AU}$, is well within the stability region. The perturbation of the singleton induces Kozai
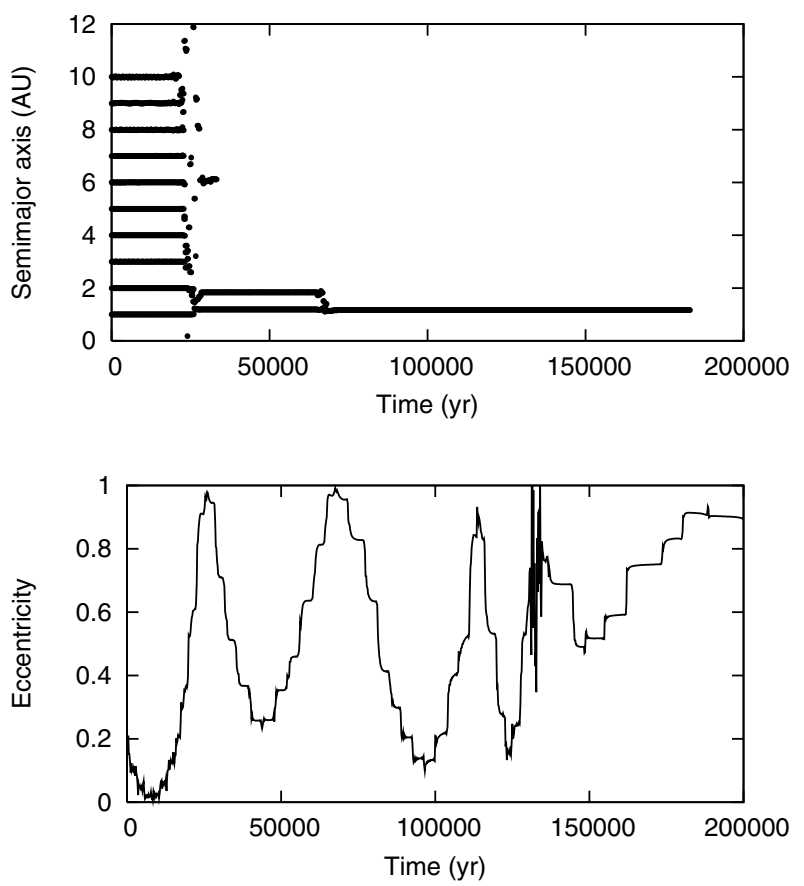

Fig. 1. Orbital evolution of the planets around the primary star of the binary (plot a) under the perturbations of the companion star, in turn affected by the gravitational pull of the outer singleton star (plot b). The initial semimajor axis of the binary is $70 \mathrm{AU}$, the eccentricity of the binary 0.2 , that of the singleton 0.2 , and the mutual inclination $i_{\mathrm{m}}$ is set to $90^{\circ}$.

cycles on the binary companion that achieves an eccentricity of almost one over a timescale of $2.5 \times 10^{4} \mathrm{yrs}$. This behaviour is well described by quadrupole and octupole-level secular equations presented in Mazeh \& Shahan (1979); Ford et al. (2000). All the planets beyond 2 AU are ejected from the system after the first cycle, while that orbiting at $2 \mathrm{AU}$ is destabilized after the second cycle. Starting from $1 \times 10^{5}$ yrs the singleton and the companion star have mutual close encounters that quickly lead to the ejection of the last inner planet, that lived through the Kozai cycles of the companion. Finally, after about $5 \times 10^{5} \mathrm{yrs}$, the outer star is ejected on a hyperbolic orbit and the the binary system is left with a smaller separation but no planets.

This kind of behaviour, typical of systems with high mutual inclination $i_{\mathrm{m}}$, places in jeopardy not only the stability of planets around the primary but also the possibility that they can form. According to Boss (1997), several gaseous protoplanets can rapidly form by disk instability in a marginally gravitationally unstable protoplanetary disk. Within this scenario in a few hundreds years we might witness the formation of the unstable triple and of a planetary system made of gas giant planets around the primary before the Kozai cycle increases the eccentricity for the companion star. On the other hand, core-accretion would not have enough time to accumulate a core by planetesimal accretion, and even planetesimals may have failed to form on such a short timescale. A protoplanetary disk around the primary star would be strongly perturbed and almost fully destroyed during the first Kozai cycle in eccentricity and inclination of the companion star. However, if the binary system was isolated during its formation and it became part of an unstable triple later on because of repeated stellar encounters in a dense star-forming region, then planets might have the time to grow even by coreaccretion, before the onset of the strong perturbations related to the stellar interactions in the triple phase. 

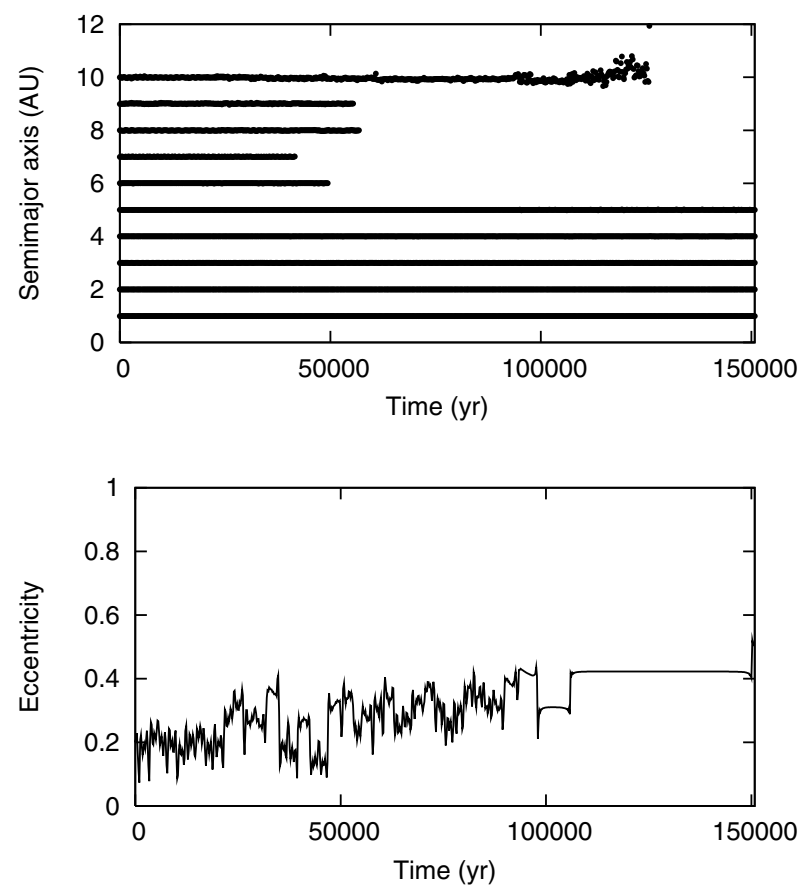

Fig. 2. Same as in Fig. 1 but for a lower value of $i_{\mathrm{m}}$ set to $30^{\circ}$.

Only systems with large values of $i_{\mathrm{m}}$ are fully destabilized by the stellar perturbations of the triple. When the mutual inclination is lower than $\sim 40^{\circ}$, close encounters between the stars, and the consequent impulsive changes of the orbital elements, are a source of instability for the planets but often not strong enough to destabilize the full planetary system. In Fig. 2 we illustrate the evolution of a model with $a_{\mathrm{b}}=70 \mathrm{AU}, a_{\mathrm{s}}=212 \mathrm{AU}$, as in the previous case, but with a lower initial mutual inclination $i_{\mathrm{m}}=30^{\circ}$. The triple quickly becomes unstable and the singleton has frequent close approaches with the binary companion marked by sudden steps in eccentricity and semimajor axis. The changes in the orbital elements of the companion leads to unstable planetary orbits as shown in Fig. 2. However, contrary to the case shown in Fig. 1 the planetary system is not fully destroyed and planets within $5 \mathrm{AU}$ of the star survive the chaotic phase. Further perturbations by the binary companion after the triple disruption do not destabilize the planetary survivors since they are well within the critical semimajor axis for stable orbits (Holman \& Wiegert 1999).

If the companion and the singleton are on retrograde orbits, instability builds up in a similar way. For mutual inclinations lower than $\sim 140^{\circ}$, large amplitude oscillations of the eccentricity begin to destabilize the planetary system which is finally destroyed by the stellar encounters in the chaotic phase. Contrary to the prograde case, the oscillations of eccentricity and inclination are not in phase, as predicted by the quadrupole theory. Apparently, the two orbital parameters are no longer bound in an invariant and they evolve with independent frequencies. For inclinations in between $\sim 140^{\circ}$ and $\sim 180^{\circ}$ the oscillations in eccentricity are moderate to low but some instability of planetary orbits is driven by the large inclination oscillations of the companion. In Fig. 3 we show the evolution of the inclinations of the singleton and companion star when the mutual initial inclination is $\sim 150^{\circ}$. We plot the inclination of each individual star, referred to the initial plane of the binary, because this is the plane where the planets also begin to orbit the primary. The inclination of the companion becomes very high and becomes retrograde for
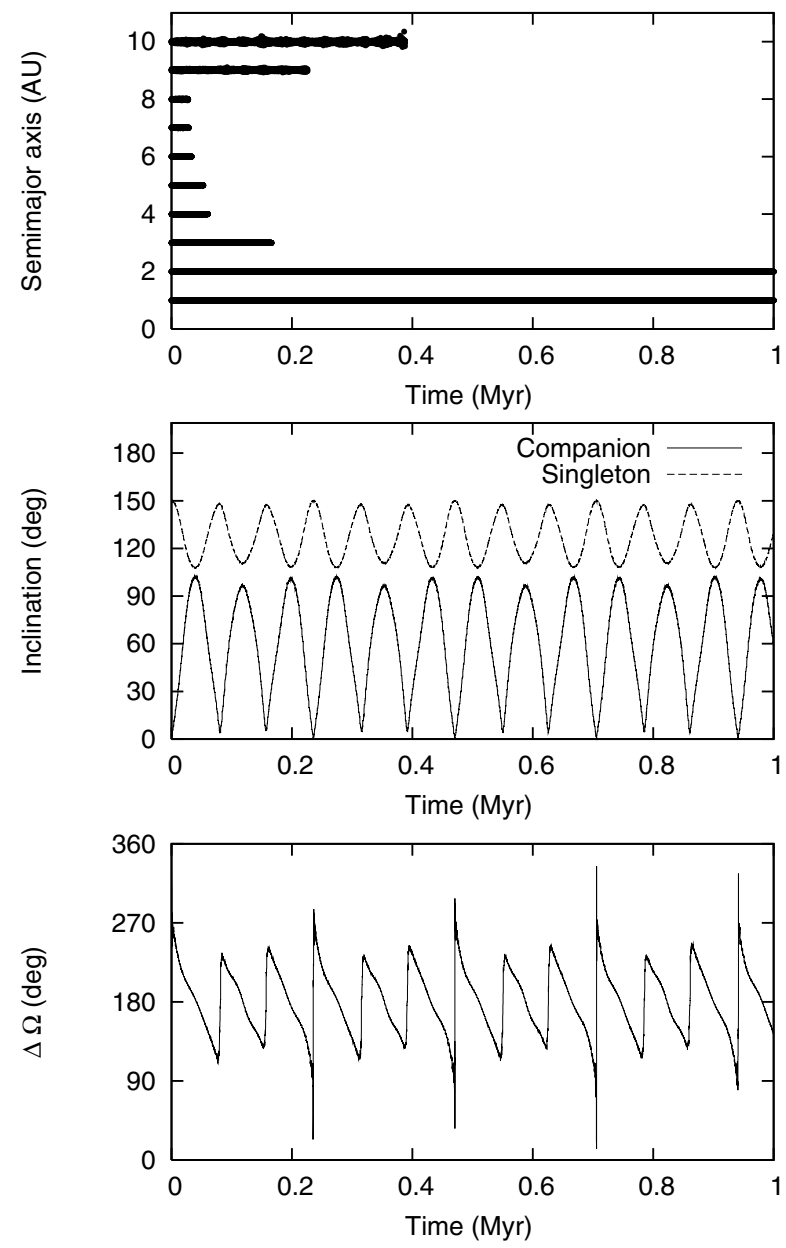

Fig. 3. Destabilization of planets around the primary when the singleton is on a retrograde orbit relative to the companion. The initial mutual inclination between the two outer stars is $150^{\circ}$. The $\Delta \Omega$ angle librates around $180^{\circ}$ while the inclinations of the two planets have wide oscillations. Only two planets, the closer ones, survive after $1 \mathrm{Myr}$ of evolution.

a short while. The behaviour is characterized also by the libration of the angle $\Omega_{2}-\Omega_{1}$ (see Fig. 3, lower panel) with the same frequency as the inclination oscillations. Most of the planetary orbits are destabilized during these large inclination excursions of the companion star and after 1 Myr only the two inner planets survive. The onset of the chaotic phase of the stars ejects finally also these two survivors.

\section{Statistical outcome}

To test the chances of a planetary system in a binary to survive a period of stellar interactions typical of an unstable triple, we have run several models with the binary semimajor axis fixed to $a_{\mathrm{b}}=70 \mathrm{AU}$. The orbital eccentricities of the stars are both set to 0.2 . In Fig. 4 we plot the percentage $P_{\mathrm{s}}$ of dynamical systems that, at the end of the period as a hierarchical triple, retain at least one of the initial 10 planets vs. $i_{\mathrm{m}}$, the initial mutual inclination between the two outer stars. This percentage is very high for low inclinations confirming the results presented in MB1 for low-inclination systems, while it declines very quickly when the inclination approaches $90^{\circ}$. This is a consequence of both the Kozai cycle that pushes the binary companion closer to the planets, and of the more complex orbital behaviour during close approaches between the stars when their orbits are inclined. 


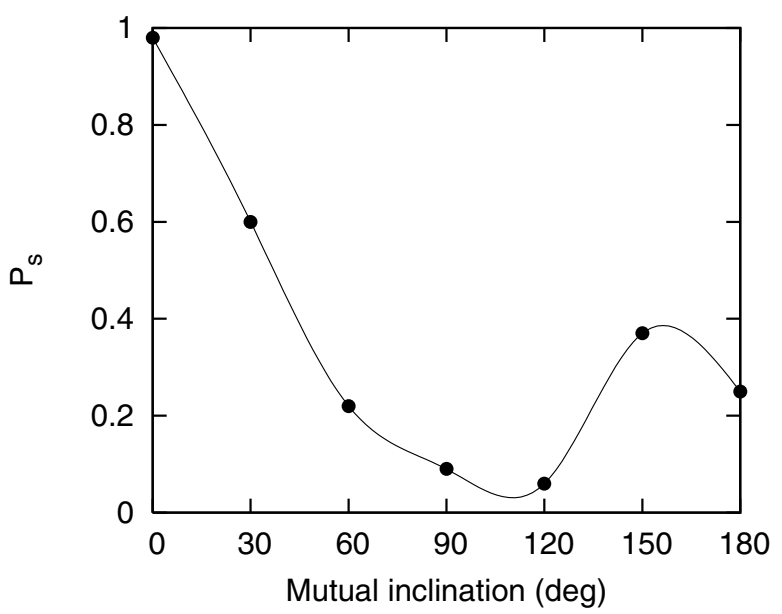

Fig. 4. Fraction $P_{\mathrm{s}}$ of binaries that, at the end of the temporary triple phase, retain at least one of the planets in a stable orbit around the primary vs. $i_{\mathrm{m}}$, the initial mutual inclination between the singleton and the binary companion.

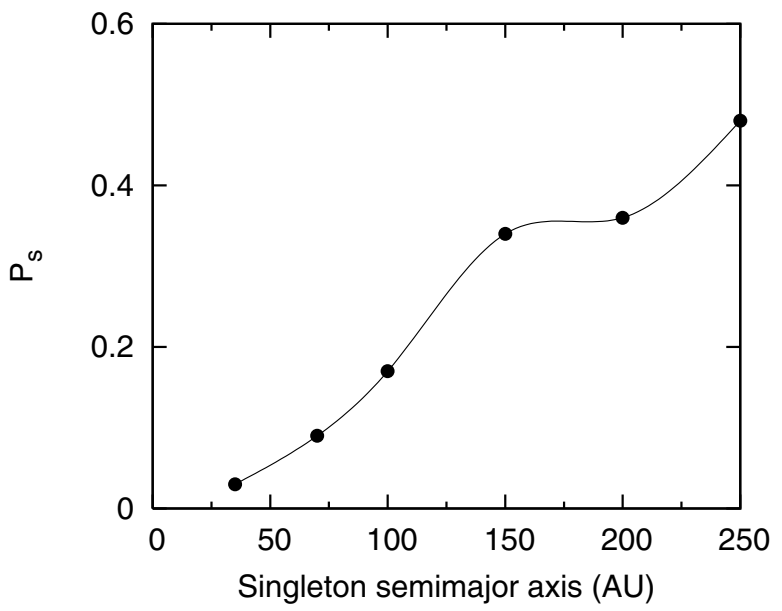

Fig. 5. Fraction $P_{\mathrm{s}}$ of binaries that, at the end of the temporary triple phase, retain at least one of the planets in a stable orbit around the primary vs. $a_{\mathrm{b}}$, the binary semimajor axis. The mutual inclination $i_{\mathrm{m}}$ is set to $90^{\circ}$, the worst case for planet stability.

Retrograde orbits of the singleton also lead to fast instability of the planets when the mutual inclination is close to $90^{\circ}$. Wide oscillations of the eccentricity up to large values are observed, even if not related to the known Kozai type mechanism: there is no phasing between eccentricity and inclination. However, even in this case when the eccentricity is at its peak value most of the planets are destabilized. Only when the mutual inclination has values beyond $\sim 140^{\circ}$ the planets around the primary are partly spared by the oscillations in eccentricity of the binary companion. However, as observed in the previous section, for mutual inclinations in the range $\sim 140^{\circ}-180^{\circ}$ large inclination oscillations of the companion star destabilize planets even if to a lesser extent than the eccentricity oscillation. As a result, the percentage of planets surviving the chaotic phase grows for inclinations larger than $90^{\circ}$ but it does not return to $100 \%$, halting at about $30 \%$.

If we increase the semimajor axis of the binary $a_{\mathrm{b}}$, the fraction of systems with surviving planets increases in an almost linear way. In Fig. 5 we show the fraction of systems retaining planets vs. $a_{\mathrm{b}}$ for the worst case, i.e. with mutual inclination equal to $90^{\circ}$. The triple instability is a mechanism that easily destroys planetary systems of close binaries while it is less effective for wide binaries. For larger values of $a_{\mathrm{b}}$ the planetary systems that survive are also more extended in semimajor axis. In most cases for $a_{\mathrm{b}}=250 \mathrm{AU}$ all the planets up to $a_{\mathrm{p}}=10 \mathrm{AU}$ survive the stellar chaotic phase.

\section{Conclusions}

The fraction of binary systems hosting planets in S-type orbits can be lower than expected. If the binary is part of a crowded stellar environment, encounters with other stellar objects can lead to the formation of a transitional triple with large mutual inclination between the singleton and the binary. The subsequent dynamic evolution of the triple, in particular the large oscillations in eccentricity of the companion star in the binary and the chaotic evolution during the triple destruction, destabilize planetary orbits around the main star. Even if the binary was born as part of an unstable inclined triple, the planetary system is fated to be disrupted.

Observing a binary system without planets in S-type orbits does not necessarily imply that the stars did not posses circumstellar disks in their early phases or that planet formation did not occur. The history of the binary and of its primordial environment must be taken into account since in most cases it may be the cause of the absence of planets. Planet formation might be a very efficient process also in the presence of external perturbations, but the survival of planetary systems may be threatened by the binary dynamical history.

Acknowledgements. We thank P. Eggleton for stimulating us to perform this work.

\section{References}

Beust, H. 2003, A\&A, 400, 1129

Boss, A. 2006, ApJ, 641, 1148

Boss, A. P. 1988, Comments Astrophy., 12, 169

Boss, A. P. 1997, Science, 276, 1836

Chambers, J. E., Quintana, E. V., Duncan, M. J., et al., AJ, 123, 2884

dalla Stella, A., Marzari, F., Barbieri, M., et al. 2005, 36th LPSC 2005, 1253

Desidera, S., \& Barbieri, M. 2007, A\&A, 462, 345

Duchene, G. 1999, A\&A, 248, 485

Duncan, M. J., Levison, H. F., \& Lee, M. H. 1998, AJ, 116, 2067

Eggleton, P., \& Kiseleva, L. 1995, ApJ, 455, 640

Everhart E. 1985, in Dynamics of comets: their origin and evolution, ed. A.

Carusi, \& G. B. Valsecchi (Dordrecht: Reidel), Proc. IAU Coll., 83, 185

Fekel, F. C. 1981, ApJ, 246, 879

Ford, E. B., Kozinsky, B., \& Rasio, F. A. 2000, ApJ, 535, 385

Ghez, A. M., Neugebauer, G., \& Matthews, K. 1993, AJ, 106, 2005

Heggie, D. C. 1975, MNRAS, 283, 566

Holman, M. J., \& Wiegert, P. A. 1999, ApJ, 117, 621

Kiseleva, L., Aarseth, S., Eggleton, P., \& de al Fuente Marcos, R. 1996, ASP Conf., Ser., 90, 433

Kohler, R. \& Leinert, C. 1998, A\&A, 331, 977

Kroupa, P. 1995, MNRAS, 277, 1491

Larson, R. B. 2001, in IAU Symp. 200, ed. H. Zinnecker \& R. D. Mathieu

Malmberg, D., Davies, M. B., \& Chambers, J. E. 2007, MNRAS, 377, L1

Marzari, F., \& Barbieri, M. 2007, A\&A, in press

Marzari, F. \& Weidenschilling, S. J. 2002, Icarus, 156, 570

Marzari, F., Weidenschilling, S. J., Barbieri, M., \& Granata, V. 2005, ApJ, 618, 502

Mazeh, T., \& Shahan, J. 1979, A\&A, 77, 145

McMillan, S., Hut, P., \& Makino, J. 1991, Ap.J., 372, 111

Muterspaugh, M. W., Lane, B. F., Konacki, M., et al. 2006, A\&A, 446, 723

Pollack, J. B., Hubickyj, O., Bodenheimer, P., et al. 1996, Icarus, 124, 62

Rasio, F. A., \& Ford, E. B. 1996, Science, 274, 954

Reipurth, B., \& Zinnecker, H. 1993, A\&A, 278, 81

Reipurth, B. 2000, AJ, 120, 3177

Sterzik, M. F., \& Tokovinin, A. A. 2002, A\&A, 384, 1030

Thébault, P., Marzari, F., Scholl, H., Turrini, D., \& Barbieri, M. 2004, A\&A, 427, 1097

Thébault, P., Marzari, F., \& Scholl, H. 2006, Icarus, 183, 193

Weidenschilling, S. J., \& Marzari, F. 1996, Nature, 384, 619 